\title{
Die Nabelschau
}

\author{
Erhard Taverna
}

Das Gifttier vom Baum der Erkenntnis hat sich in eine lange Warteschlange verwandelt. Wer den Eingang zum Garten Eden endlich durchschritten hat, hört Vögel zwitschern und erblickt als erstes den berühmten Baum. Farbig leuchten seine elektronisch bewegten Blätter an der Spiegelwand im rot-gelb-grün-blauen Scheinwerferlicht. Das Paradies der Firma Roche gleicht einem Dancing, das die Besucher mit dekorativen Windrädern, interaktiven Computerspielen und in Grashügeln versteckten Minibühnen unterhält. Hier zielen die Verbote vor allem auf das Übergewicht und seine bösen Folgen. Vorläufig leiden wir noch unter der steinzeitlichen Erblast, doch die frohe Botschaft kündigt sich an in den von ihren Übeln zu reinigenden Chromosomen. Auch Novartis ist im Reich der Mythen fündig geworden und belohnt die Harrenden mit einem Jungbrunnen im Biopolis des Jahres 2022. Am Brunnenrand wandern farbige Blasen über berührungssensible Bildschirme, die durch Antippen balanciert werden wollen. Während sich die Besucher abmühen, schwimmen Adam und Eva munter durch das Wunderwasser. Fernsehgesichter reden von Lifestyleberatung und Kreislaufchecks durch Putzroboter, und wer in Nischen Platz nimmt, den berieselt die futuristische Werbung. Man weiss nicht, was erstaunlicher ist, dass Biopolis immer noch Deutsch spricht oder die nächste Generation der Chromosomeningenieure und Nanotechniker das Geheimnis des Alterns geknackt hat.

Lifescience und Medizin sind im Dauertrend, und der Auftrag ist klar. Die «feel good»-Tage der Migros sind unsere Zukunft. Coop liefert das Manna in Neuchâtel, wo fit und munter im Schlaraffenland lebt, wer in den richtigen Bioapfel beisst. Die Sorge um das eigene Wohl ist ein zentrales Thema der Arteplages, die in spielerischer Form die Kulissen des Glücks inszenieren. Die frei wählbaren Kinosäle in «Swisslove» versprechen Alltagsgeschichten vor romantischer Bündner Kulisse und erzählen die Beziehungskisten von drei Generationen im Stil der bekannten Soap Opera von «Lüthi und Blanc». Im «Le premier regard» geht es um die sexuellen Einladungen der Köpersignale, Blickkontakte und Duftstoffe. Balzgefieder, röhrende Hirsche und Trüffelpheromone führen zum Ausgang, wo das BAG zum Kondomgebrauch ermahnt. Im Pavillon «Wer bin ich?» liegen die Besucher im Halb-

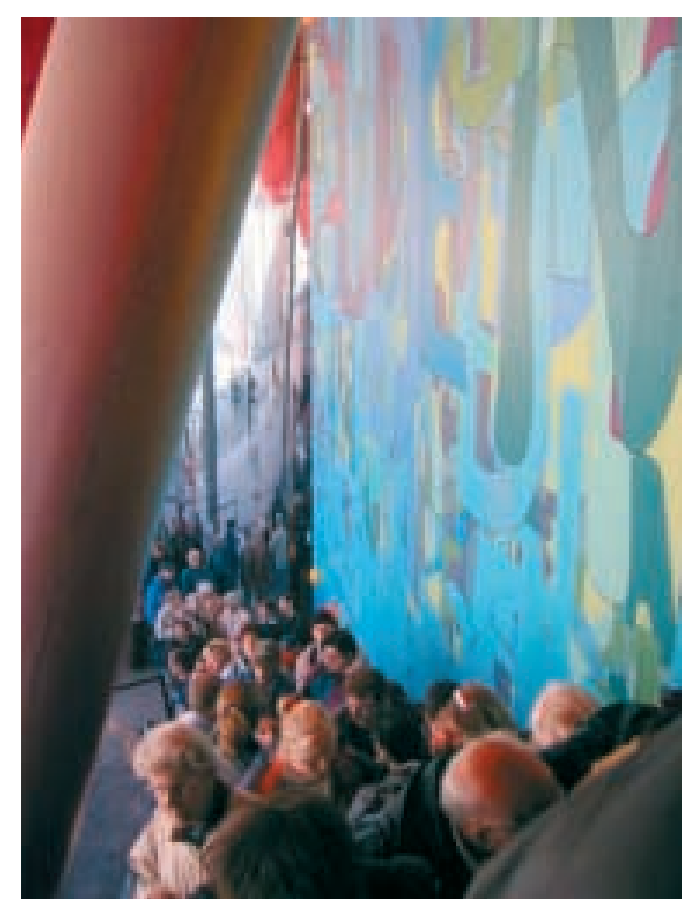

dunkel auf bequem weichen Liegen und suchen ihr Ego an der wolkigen Filmdecke, während andere im «Happy End» den Glückssinn entdecken und über eine Rutschbahn ins Freie gelangen. Die Formen des Unglücks wie Krankheit, Armut, Ohnmacht und Zerstörung der Umwelt verschwinden fast ganz in der Abstraktion. Sie gerinnen zur Statistik, werden zum neckischen Schlauchlabyrinth im «Signalschmerz», schrumpfen zu Strichfigürchen im «Garten der Gewalt» oder sind schönes Lichtspiel auf einem rotierenden Globus im «Palais de l'Equilibre». Die Politik muss auf der «Werft» in Murten aus der Europanto-Sprache und den Symbolen der Laufportale übersetzt werden oder wird in Biels «Nouvelle DestiNation» zum Sport versimpelt.

Die Tinguely-Maschine von 1964 hat sich durchgesetzt. Es gab viele brillante Einfälle mit einer perfekten Bildsprache zu bewundern. Die Architektur war elegant und phantasievoll, das viele Personal freundlich und die Organisation hervorragend. Und wenn es auch viele nicht wahrhaben wollen: die Inszenierungen waren präzise Projektionsflächen für die Gefühle, Wünsche und Hoffnungen der Nach-Landi-Generationen. In unserer multioptionalen Gesellschaft ist jeder seines Glückes Schmied, muss sich jeder und jede unablässig selber erfinden. Dieser Auf- 
gabe entsprach der Expo-Patriotismus light. Er war friedlich wie eine Love Parade. Das «heimliche Imperium» von einst mochte nicht mehr bis an die Zähne bewaffnet sein. Oder mit den Worten von Frau Ruth Dreifuss: "On voit les drapeaux sur chaque cabane à outils. C'est une image de la Suisse très belle. Mais une exposition nationale n'est pas compatible aux jardins familiaux.» Ein Bauwerk hat dem Zeitgeist ein prägnantes Denkmal gesetzt. Es war der rostende Monolith im Murtensee. Die Vision einer Böcklin-Toteninsel des 21. Jahrhunderts, von der die Besucher in ihren Booten wieder zurückkommen; Biotechnik sei Dank.

\section{Wie Sitten sich wandeln}

\section{B. Gurtner}

Korrespondenz:

Dr. med. Bernhard Gurtner Eggstrasse 76

CH-8620 Wetzikon
Chefarztvisiten sind auch nicht mehr, was sie einmal waren. Als überliefertes Ritual werden sie noch zelebriert, doch ist ihre Blütezeit vorbei, das Verfalldatum naht. Bildgebende Verfahren ersetzen die umständliche Meinungsbildung am Krankenbett, computergestützte Lernprogramme verdrängen das klassische «bedside teaching». Nur in wirklichkeitsfremden TV-Schnulzen haben jene stämmigen Oberschwestern überlebt, welche in Erwartung des höchsten Herrn mit Feldweibelstimme alle Patienten in ihre Betten befehlen und die frischen Leintücher straff strecken lassen. Die heilbringende Prozession führt heute nicht mehr zu unterwürfigen Schlitzhemden, sondern trifft auf kritische Spitalkunden, sofern sich diese nicht im eleganten Sportdress auf den Raucherbalkon oder in die Cafeteria verzogen haben.

Nach wie vor werden aber die Bettlägerigen von einem grossen Tross im Halbkreis bedrängt, sehen sich entblösst von zahlreichen Blicken durchbohrt und kommen kaum zu Wort. Befunde sind wichtiger als das Befinden. In der hintersten Reihe schäkern die Medizinstudenten mit den Pflegeschülerinnen und im vordersten Glied vertiefen sich Chefarzt und Oberarzt in die vom Assistenten herausgeklaubten Laborberichte oder wenden sich rechtsdrehend einem gegen das Tageslicht hochgehaltenen Röntgenbild zu, auf dem die Heimgesuchten schaudernd von ferne ihr Skelett erahnen können. In modern aufgerüsteten Kliniken werden Patientendaten elektronisch abgerufen und wenigen Einsichtigen in einem beschränkten PDA-Blickfeld hingepixelt. Die weiter hinten Stehenden lassen sich nicht ungern zu interessanteren Tätigkeiten wegrufen, das Gefolge verdünnt sich fortlaufend von Zimmer zu Zimmer.
Internisten, Psychiater, Soziologen, Kommunikationsforscher und Pflegewissenschafterinnen haben die Chefarztvisite in zahlreichen Studien untersucht. Ihre Beobachtungen dürften den jeweiligen Ablauf ähnlich beeinflusst haben wie physikalische Experimente das Verhalten von Elementarteilchen. Walter Vogt hat als Insider in seinem «Wüthrich» (1966) die dreifach wiederholte Inspektionsrunde eines moribunden Klinikchefs so gnadenlos dargestellt, dass ihn einige Kollegen aus der FMH ausstossen wollten. Solche Übertreibungen stammen aber aus der uns allen gemeinsamen Erfahrung, dass sich Visiten kaum je in einer allseits befriedigenden Form gestalten lassen.

Im Idealfall könnten die Erkrankten zuerst ihre Beschwerden und Befürchtungen schildern, ohne schon nach wenigen Sekunden unterbrochen zu werden. Darauf würden sie unter möglichster Schonung ihrer Privatsphäre vom Chefarzt ergänzend befragt und persönlich untersucht. Der Assistent müsste über Anamnese, bisherige Befunde, Behandlung und Verlauf zusammenfassend berichten. Das Pflegepersonal sollte eigene Beobachtungen und Anregungen beisteuern. Die Medizinstudentin im Praktikum dürfte die vergrösserte Milz palpieren und würde als Lernende mit einer Frage zur Pathophysiologie bedacht, um unwissende Assistenten zu schulen, ohne sie vor allen Anwesenden zu blamieren. Dann kämen die begleitende Physiotherapeutin oder Ernährungsberaterin zu Wort. Abschliessend würde das weitere Vorgehen gemeinsam in verständlicher Sprache festgelegt und nochmals Gelegenheit zu offenen Fragen geboten. Alle Entscheide wären transparent, kostenbewusst und EBM-konform. Im Korridor würden die wartenden Angehörigen vom Chef ausführlichst über Diagnose und Pro- 
gnose informiert, wobei der Oberarzt Gelegenheit fände, dem Pflegepersonal tuschelnd zu erklären, was schlussendlich zu tun sei ...

$\mathrm{Na}$ ja. Wenn in knapper Zeit viele Patienten besucht werden müssen, fällt von diesem (internistischen) Maximalprogramm einiges weg. Dazu kommen unschöne Begleiterscheinungen der Visiten, die wir als geknutete Heilknechte selbst zu erdulden hatten und später als Kaderärzte den anderen zumuteten. Der Frust unzulänglicher Kontakte lässt sich aber durch einige Gewohnheiten mildern, die man sich schon als Assistent(in) aneignen kann:

- Reichen Sie den Patienten bei der Begrüssung die Hand, auch wenn es die Hygienebeauftragten nicht schätzen. Wenn Sie zudem den Puls fühlen, spüren Sie hautnah, wie es einem Kranken geht.
- Setzen Sie sich seitlich ans Bett, selbst wenn Sie nur wenig Zeit haben und erst einen Stuhl heranrücken müssen. Eine Minute Augenkontakt und ein Dialog auf gleicher Höhe bringen oft mehr, als wenn Sie von oben herab ein fünfminütiges Ferngespräch führen, bei dem Sie sich hinter der Fussleiste des Bettes inmitten einer weissen Leibgarde aufgestellt haben.

- Widmen Sie allen Kranken in einem Zimmer ungefähr gleich viel Aufmerksamkeit. Bei Langzeithospitalisierten geht es einmal rechts herum, das nächste Mal links herum, damit nicht immer Frau Müller zuerst an der Reihe ist.

- Kommen Sie nach der pünktlich begonnenen und fix beendeten Visite nochmals zurück, wenn jemand längere Zuwendung braucht oder machen Sie dort am Abend ganz allein eine «Bettmümpfelivisite». Es wird auch Ihnen guttun.

\title{
«Macht Scham depressiv?»
}

\author{
Gedanken zu einem Vortrag von Prof. Dr. med. D. Hell*
}

R. Schild

* gehalten am 25. Januar 2002 in der Psychiatrischen Universitätsklinik Burghölzli, Zürich.

Korrespondenz:

Dr. med. Rolf Schild

Sengglenstrasse 8

CH-8118 Pfaffhausen
Das Thema und seine Darstellung durch D. Hell haben mich fasziniert, der Titel hat mich provoziert. Hell versucht, mit einer auch für NichtPsychiater verständlichen Sprache, die verschiedenen Facetten der Scham und ihre Beziehung zur Depression einem weiteren Kollegenkreis für Diagnostik und Therapie näherzubringen. Die vielleicht gewollte Einseitigkeit des Titels macht mich neugierig.

Viele - die meisten (oder alle?) - depressiven Menschen schämen sich ihrer Stimmungsstörung, insbesondere aber deren Folgen. Hell weist in seinem Vortrag auf verschiedenartige Herkunft, als auch Herkunftsweisen hin. Schon allein der Hinweis auf Scham als sogenannten «sozialen Affekt» (s. auch bei: Pfau, Tisseront und Wurmser) auch ohne (manifeste) Depression, scheint uns in der heutigen Zeit der weit verbreiteten narzisstischen Verletzungen, insbesondere im Verhältnis Arzt-Patient-Arzt, Patient-Arzt-Patient, von eminenter Bedeutung. Hell unterscheidet in eindeutiger Weise «Beschämung», d.h. passiv von aussen in Frage gestellt, vom autonomen «Schamempfinden», d.h., «ich schäme mich», ich stelle mich selbst in Frage. Diese Abgrenzung nach Verursachung ruft nach weiteren Fragen. Zunächst bewirkt die verschiedene Verursachung dieselben qualitativ oder/und quantitativen Empfindungen oder grenzen sich diese voneinander $a b$ ? (Scham der «Beschämung» und Scham von «sich schämen»). Es liesse sich auf die Titelfrage eine Zusatzfrage stellen: welche der beiden oder auch beide Erscheinungsbilder der Scham machen depressiv? In dem von Hell in seinem Vortrag beschriebenen Verlauf bei einem Patienten scheint die Beschämung ausschlaggebend gewesen zu sein. Es liesse sich auch überlegen, wieweit eine vorbestehende Schamhaftigkeit für die Verarbeitung einer Beschämung eine Rolle spielen kann. Dabei kommt es auch zum Diskurs, dass Schamfähigkeit, worauf auch Hell hinweist, im «Sich-schämen-Können» als eine erwünschte Eigenschaft betrachtet wird. So z.B. als Fehlen «schamlosen» Verhaltens. Diese «selbst-gerechte» 
Fähigkeit (bei Hell: Selbsterkenntnis und Selbstbewusstsein) kann aber nur von relativ Ich-starken Personen erwartet werden, nicht aber von Depressiven, die, wie Hell in äusserst treffender Sprache sagt, bereits unter sogenannten «-losigkeiten» leiden (freud-, energie-, antriebs-, ideen-, entscheidungs-, interesse-, appetit-, schlaf- und lustlos). Das «Sich-in-Fragestellen» im Schameffekt erscheint wie eine Art Komprimierung der Aspekte der Depression, also eben dieser «-losigkeiten». Mit dieser Sicht ist Scham weder Ursache noch Wirkung einer Depression, sondern eine ihrer Ausdrucksmöglichkeiten. Dies gilt sowohl für die Wirkung durch Beschämung als auch für das eigenständige Schamempfinden in einer Depression (vor, in und nach) und als Folge narzisstischer Kränkung und Verletzung durch die Akzeptanz somatischen, aber insbesondere psychischen Krankseins. Die «Alltagsscham» könnte so der Möglichkeit von Trauerarbeit entsprechen. In einer echten Depression ist Trauerarbeit nicht mehr möglich, was heissen würde, dass in der Depression zunächst auch kein Schamgefühl zugelassen werden kann, somit zur tiefen Depression auch der Verlust des Schamgefühls gehört. Es wäre dies eine «Scham-losigkeit» im Sinne von Hells depressiver Reihe von «-losigkeiten». Wieweit diese -losigkeit im Sinne des üblichen Sprachgebrauchs im subjektiven Empfinden eines depressiven Patienten eine Rolle spielt («ich bin ein schlechter Mensch - ich schäme mich»), könnte für das therapeutische Angehen der Schamproblematik und der Depression von Bedeutung sein. Das Auftauchen einer Schamthematik wird damit zu einem Indikator eines Fortschritts in der Therapie.

Nach Hell kann der Zugang zu Schamgefühlen für den Patienten erleichtert werden, wenn aufkommende unangenehme Gefühle, wie Wut, Ekel oder Angst, vom Therapeuten verstanden werden und vom Patienten dann auch zugelassen werden können. Im Kontext erscheint dies wie eine Bahnung für ein allmähliches Zulassen von «Scham-Angst» und «Scham vor der Scham». Dieses Vorgehen soll in «sanften» Schritten vor sich gehen. Nach Hell stellt die «Akzeptanz» der sogenannten unangenehmen Gefühle auch ein Gegengewicht zur depressiven Leere (-losigkeiten?) dar. Eine solche Akzeptanz sei jedoch erst möglich, wenn die depressive Blockade akzeptiert sei. Ich meinerseits würde lieber sagen «an-erkannt» werde, da Akzeptanz eine Fähigkeit darstellt, die von einem depressiven Patienten noch nicht erwartet werden kann (Fehlen der dazu nötigen Souveränität und des dazugehörigen Selbstwertgefühls). Hell sagt auch: «Scham ist im alten Testament das erste Gefühl, das aus- drücklich beim Namen genannt wird. Adam und Eva schämen sich, vom Baum der Erkenntnis gegessen zu haben. Scham hat nach der Schöpfungsgeschichte der Bibel also mit Selbsterkenntnis zu tun.» (s. oben: «selbstgerecht»). Das Auftreten der Selbsterkenntnis beim Kleinkind im Spiegel (12-15 Monate) stellt die Vorbedingungen für die Entwicklung der Scham (mit etwa 2-3 Jahren. Darwin zit. n. Hell).

Es liesse sich nun fragen: Hat die Selbsterkenntnis zur Vertreibung aus dem Paradies geführt, oder ist es umgekehrt, hat die Vertreibung aus dem Paradies die Selbsterkenntnis erst ermöglicht? So oder so, nicht unbedingt eine müssige Frage, finden wir doch dieselbe Problematik für das Auftreten von Scham bei der Entwicklung des Kindes: die ersten 2-3 Jahre sind noch relativ paradiesisch - selbstbezogen, narzisstisch stark in der Einheit mit der Umwelt. Je mehr Trennung und Gegensatz zur Umwelt, desto mehr realisiert das Kind, etwas Eigenständiges, aber damit auch gegenüber der Umwelt etwas Kontroverses zu sein. Erst mit der Abgrenzung und der Anerkennung eines sogenannten «anderen», d.h. Existenz von Objekten (im analytischen Sinne) ausserhalb der eigenen Existenz, kann ein Kind schuldfähig werden und damit auch schamfähig. Die christliche Kunst stellt Eva und Adam nackt dar. Paradies heisst demnach «Nacktsein» können und dürfen, wir würden sagen, auch seelisch-geistig nackt und, weil paradiesisch, unverletzbar. Erst die Vertreibung aus dem Paradies bringt die (narzisstische) Verletzbarkeit und damit die Notwendigkeit eines Schutzes in Form einer Maske (Feigenblatt) und damit auch die Möglichkeit von Scham. Der Schamaffekt (Feigenblattmaske) versucht mit seiner Funktion als Maske der Maske die entstandene Verletzbarkeit zumindest zu lindern. Damit erhält auch das «Sich-schämen-Können» eine Dimension der Ich-Stärke.

Als therapeutisches Ziel heisst das: Möglichkeit zur Anerkennung eines Versagens und sich dessen schämen können und dürfen - als positive Fähigkeit, zu erkennen und anzuerkennen. In der etymologischen und sprachspezifischen Literatur wird immer wieder auf die Bipolarität des Schambegriffs hingewiesen. Einerseits ist die Scham Ausdruck schlimmster, unangenehmer Gefühle der Peinlichkeit, der Schmach und Schande und des Hohns. Andererseits ist sie eine Tugend mit hemmender Funktion für falsches Handeln. Es sind dies zwei verschiedene Ausdrucksformen und Auswirkungen des Schamgefühls. Diese Dichotomie des Schambegriffs kommt in verschiedenen Sprachen durch verschiedene Worte zum Ausdruck. Im Gegensatz 
zu den germanischen Sprachen, die nur ein «einheimisches» Wort zur Bezeichnung des komplexen Schamgefühls besitzen, kennen das Griechische, Lateinische und Französische mehrere Worte, die jedoch in jeder Sprache jeweilen wieder andere, nicht vergleichbare Bedeutungsinhalte haben (zum Teil zit. nach Yeandle). So gibt es im Französischen zwei Worte für Scham: «honte», meist in pejorativem Sinne wie Schande, daneben aber auch neutraler als Schamhaftigkeit. Demgegenüber steht «pudeur» für Schamhaftigkeit, Züchtigkeit, Verschämtheit und damit schamlos: «sans pudeur».

Diese linguistisch-etymologischen Überlegungen möchten einen Beitrag leisten für ein adäquateres Verständnis im Umgang mit Patienten anderer Kulturen und Sprachen. In diesem Zusammenhang wollen wir Hell zitieren, «dass Scham ein Gefühl ist, das soziale Vorgänge differenziert wiedergibt» und «ein Selbstbewusstsein voraussetzt». Hell weist damit auf den der Scham beigemessenen Wert in einer jeweiligen Gesellschaft. Margaret Mead hat 1937 aufgrund ihrer Forschungsarbeiten «Schamkulturen» und «Schuldkulturen» unterschieden. Schamkulturen sind besonders unter Naturvölkern vertreten, Schuldkulturen eher unter Gesellschaften «weiterentwickelter Religionen». (z. B. Christentum). Es können jedoch innerhalb derselben Gesellschaften beide Kulturformen vertreten sein. Bei den Schamkulturen geht es um den öffentlichen Ruf eines Menschen und sein Bestreben, diesen Ruf aufrechtzuerhalten und zu stärken. (zit. nach Yeandle). Nach Ward beziehen sich dagegen die Schuldkulturen auf das Seelenheil des Menschen und bieten ihm die Möglichkeit innerer Reue und öffentlicher Busse. Im Fazit seiner therapeutischen Erfahrungen, dass im «Offenlegen der Scham und im Annehmen des Schamerlebens eine befreiende Kraft stecken kann», zitiert Hell die Psychotherapeutin H. B. Lewis, die sagt, «dass Widerstand der falsche Name für Scham und Schuld ist», ein vielleicht allzu kategorischer, aber doch gezielt provozierender Hinweis. Die eigenen Schlussworte von Hell widerspiegeln die von ihm angestrebten Mittel und Ziele und deren therapeutische Atmosphäre: «Die heute aufkommende Tendenz, krankhafte von gesunden Gefühlen abzugrenzen, scheint mir gefährlich. Denn auch unangenehme Gefühle sind basale Elemente unserer Lebensorganisation. So verweisen uns Schamgefühle darauf, dass wir gedemütigt worden sind oder uns selber beschämen. Nicht das Schamempfinden ist krankhaft, sondern die Beschämung, worauf das Schamempfinden verweist, ist krankmachend. Unsere Gefühle sind nichts Zufälliges und schon gar nichts Schädliches. Es sind gerade depressive Menschen, die uns den Wert des Gemütslebens in aller Deutlichkeit vor Augen führen.»

Literaturverzeichnis beim Autor. 